\title{
An Efficient and Concise Synthesis of Heteroaryldipyrromethanes, Tetrapyrazolylporphyrins and Metalloporphyrins
}

\author{
Farhanullah and Vishnu Ji Ram*
}

Medicinal and Process Chemistry Division, Central Drug Research Institute Lucknow 226001, India

\begin{abstract}
One pot synthesis of terpyrromethanes (3a) and [di(1H-pyrrol-2-yl)methyl]heteroarenes (3b-m) has been delineated by Amberlyst 15 catalyzed condensation of pyrrole and heterocyclic aldehydes (2), not reported earlier. The compounds 3a and $\mathbf{3 b}$ have been formylated to 5-[di(5-formyl-1H-pyrrol-2-yl)methyl]- $1 H$-pyrrole-2-carbaldehyde (4a) and 5[(5-formyl-1H-pyrrol-2-yl)(3-pyridinyl)methyl]-1 $H$-pyrrole-2-carbaldehyde (4b), by using a mixture of $\mathrm{POCl}_{3}$ and $\mathrm{DMF}$ as formylating agent. Similarly, 5-alkyl-1H-pyrrole-2-carbaldehyde (8) has also been prepared from 2-alkyl-1H-pyrroles (6), obtainable by acylation followed by Wolf-Kishner reduction. Amberlyst 15 / TFA catalyzed the condensation of 8 with pyrrole which gave 3a instead of expected product 10, due to an unusual cleavage of alkyl substituent at position 5 in 8. $\mathrm{CS}_{2}$ addition to 6 followed by methylation provided methyl 5-alkyl-1H-pyrrole-2-carbodithioates (7), which on reaction with hydrazine hydrate yielded 5-alkyl-1 $H$-pyrrole-2-carbothiohydrazide (9). Tetrapyrazolylporphyrins (12) have been synthesized directly by condensation of pyrrole with 3-aryl-4-formyl-1-phenylpyrazole (11) in propionic acid. These porphyrins were transformed to metalloporphyrins (13) from reaction with zinc and copper acetate separately.
\end{abstract}

\section{INTRODUCTION}

Porphyrins represent one of the most fascinating classes of compounds because of their presence in nature and their unique physical and chemical properties. They play significant role in numerous biological systems [1] due to their coordinating ability with various transition metals to form metalloporphyrins. These compounds are currently in focus for their applications in supramolecular and material chemistry [2] owing to electrochemical, magnetic, catalytic, photochemical and photo-physical properties [3, 4]. Over the past two decades, the preparation of substituted porphyrins has attracted much attention, as they provide useful platform to introduce various functional groups with precise locations and orientations near the porphyrin backbone. The ability to anchor various groups on heterocyclic systems offers many exciting opportunities for engineering porphyrin platforms. These simple molecules have potential of useful building blocks for the preparation of heteronuclear porphyrins and the development of redox switchable tetrapyrrolic macrocycles. In the past years, numerous advances in porphyrin synthetic methodology have been realized [5]. These developments have been advanced systematically through monopyrrole tetramerization and dipyrromethane self-condensation in organic acid. The importance of photosensitizing properties [6] of these heterocycles made them useful for photodynamic therapy of tumors. This led to the synthesis of 5heteroaryldipyrromethane as new building blocks for the synthesis of porphyrins [7], expanded porphyrins and porphyrin analogs [8].

Earlier, 5-aryldipyrromethanes, used as a precursor for the synthesis of porphyrins have been synthesized [9-14] by the reaction of aldehydes with excess of pyrrole in the

*Address correspondence to this author at the Medicinal and Process Chemistry Division, Central Drug Research Institute Lucknow 226001, India; Tel: +91 522 2960779; Fax: +91 5222623 405; E-mail: vjiram@yahoo.com presence of catalytic amount of acid to suppress the side reaction of polymerization. Nagarkatti and Ashley [9] reported first the synthesis of 4-pyridyldipyrromethane by condensation of 4-pyridylcarboxaldehyde and pyrrole in acidified methanol. Later on Casiraghi et al. [10] prepared dipyrromethanes from reaction of aliphatic aldehydes with Grignard reagent, derived from pyrrole in the presence of $\mathrm{TiCl}_{4}$. Recently, a direct synthesis of substituted dipyrromethanes has been reported [15] through condensation of pyrrole with aromatic aldehydes in THF/acetic acid. In addition to the direct syntheses, two stepwise syntheses of meso substituted, $\beta$-unsubstituted dipyrromethanes have been developed $[16,17]$. We now report one pot synthesis of 5-heteroaryldipyrromethanes and terpyrromethane from Amberlyst 15 catalyzed condensation of pyrrole with 2-formylpyrrole, 3pyridylcarboxaldehyde, 1,3-diaryl-4-formylpyrazole, and 3aryl-5-formylisoxazole separately. The [di(1H-pyrrol-2yl)methyl]heteroarenes have been quite often used as precursor for the synthesis of porphyrins. Most of the porphyrin syntheses proceed by tetramerization of monopyrrole. In the classical Adler-Longo porphyrin synthesis [18], a solution of aldehyde and pyrrole in high boiling acid solvent is refluxed with air condenser so that condensation-oxidation could occur simultaneously. Lindsey et al. [19] reported the improved methodology for the preparation of compounds of this ring system. In this manuscript, we report a direct method for the preparation of porphyrins by heating a mixture of pyrrole and heterocyclic aldehydes in propionic acid without using 2,3-dichloro-5,6-dicyano-1,4-benzoquinone (DDQ) as an oxidant.

\section{RESULTS AND DISCUSSION}

5-Heteroaryldipyrromethanes have been obtained through Amberlyst 15 or TFA catalyzed condensation of heterocyclic aldehydes with pyrrole. Thus, stirring an equimolar mixture of heteroarylaldehyde (2) and pyrrole (1) using either Amberlyst 15 or catalytic amount of trifluoroacetic 


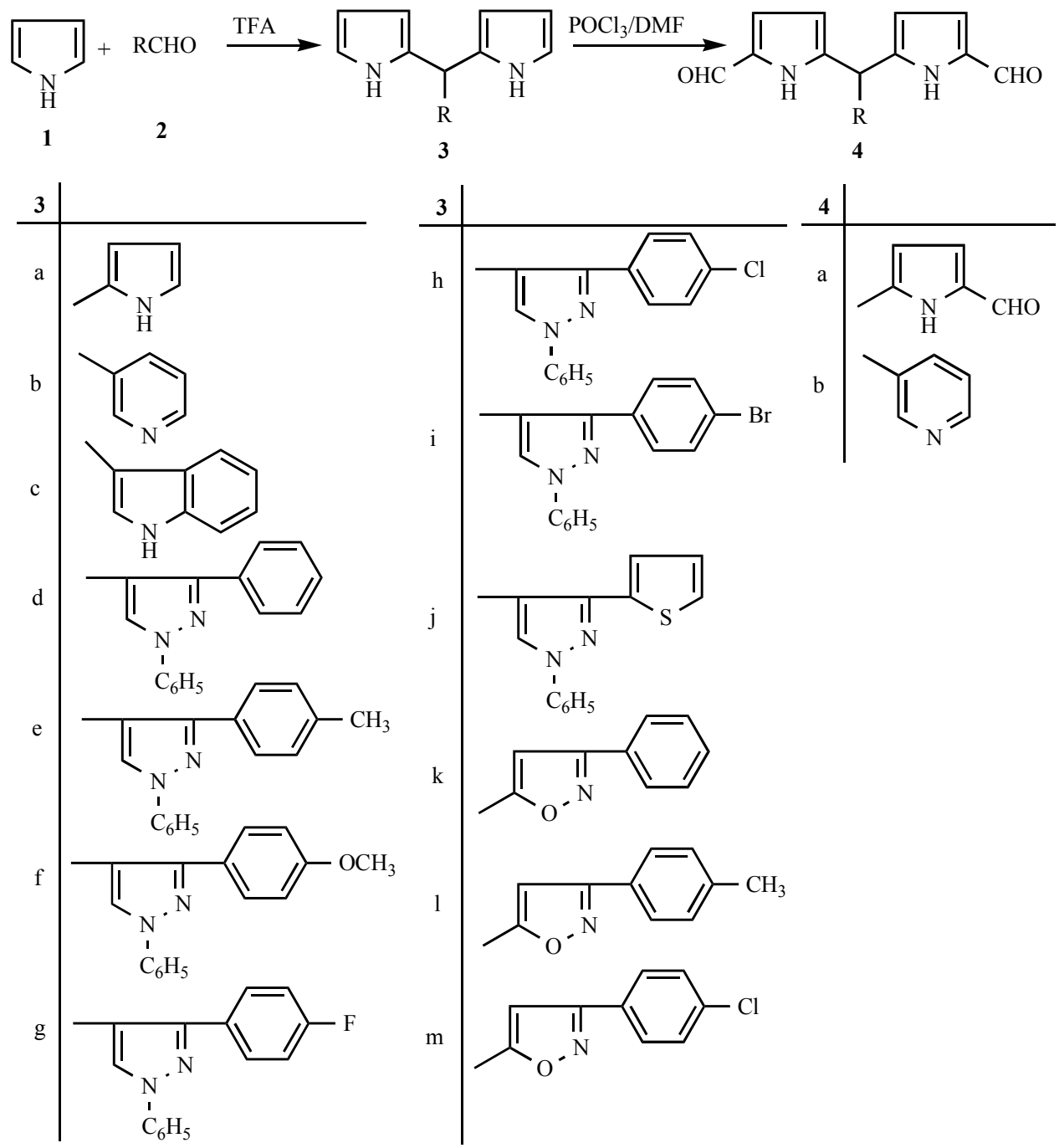

Scheme 1.

acid at room temperature for 30 minutes afforded crude [di(1H-pyrrol-2-yl)methyl]hetroarenes (3) after usual workup (Scheme 1).

The crude product obtained was purified through silica gel column using a mixture of hexane: ethyl acetate (39:1) as eluent. The Amberlyst 15 was used as a catalyst because it is environment friendly, reusable, economically viable, commercially available and convenient to handle during the reaction. These points make Amberlyst 15 superior to the other reported acid catalysts. The isolated 2-[di(1H-pyrrol-2yl)methyl]-1 $H$-pyrrole (3a) and 3-[di(1H-pyrrol-2-yl)methyl] pyridine $(\mathbf{3 b})$ were formylated to 5 -[ $\operatorname{di}(5$-formyl- $1 H$-pyrrol2-yl)methyl]-1H-pyrrole-2-carbaldehyde (4a) and 5-[(5formyl-1 $H$-pyrrol-2-yl)(3-pyridinyl)methyl]-1 $H$-pyrrole-2carbaldehyde (4b) by Vilsmeier-Haack reaction. 2-Alkyl$1 \mathrm{H}$-pyrroles (6), used as precursors for the synthesis of various pyrrole derivatives were synthesized [20] by acylation followed by Wolf-Kishner reduction. Further, a reaction of 6 with carbon disulfide in the presence of super base system $\mathrm{KOH}-\mathrm{DMSO}$, regioselectively, yielded pyrroldithiocarboxylic acid, which on methylation with methyl iodide produced methyl 5-alkyl-1H-pyrrole-2-carbodithioate (7). Compound 7 on reaction with hydrazine hydrate provided 5-alkyl- $1 H$ pyrrole-2-carbothiohydrazide (9).
The acid-catalyzed condensation of pyrrole and 5-alkyl$1 H$-pyrrole-2-carbaldehyde (8) obtained by formylation of 6 led to yield 3a instead of monoalkylterpyrromethane (10).

It was conspicuous that a reaction of 8a-e with pyrrole in the presence of TFA always produced $\mathbf{3 a}$ due to an unusual cleavage of alkyl chain present at position 5 of the pyrrole ring in 8 (Scheme 2). Porphyrins with four 1,3-diaryl-4pyrazolyl groups present at methylene junction of two pyrrole units are not reported so far. These compounds were prepared with the consideration that the presence of therapeutically important pyrazole moiety in the porphyrins unit would possibly enhance its pharmacological profiles. Attempts to prepare tetrapyrazolylporphyrin by acid-catalyzed condensation of 1,3-diaryl-4-formylpyrazole [21] and 5-(1,3diaryl-4-pyrazolyl) dipyrromethane in the presence of DDQ as an oxidant failed.

To simplify this procedure [17] an equimolar mixture of 1,3-diaryl-4-formylpyrazole $\mathbf{1 1}$ and pyrrole $\mathbf{1}$ in propionic acid was heated to $135-140^{\circ} \mathrm{C}$ for $5-6 \mathrm{~h}$ and thereafter the solution was left overnight at room temperature under nitrogen blanket. The reaction mixture was diluted with water and filtered. The solid obtained was purified on neutral alumina 


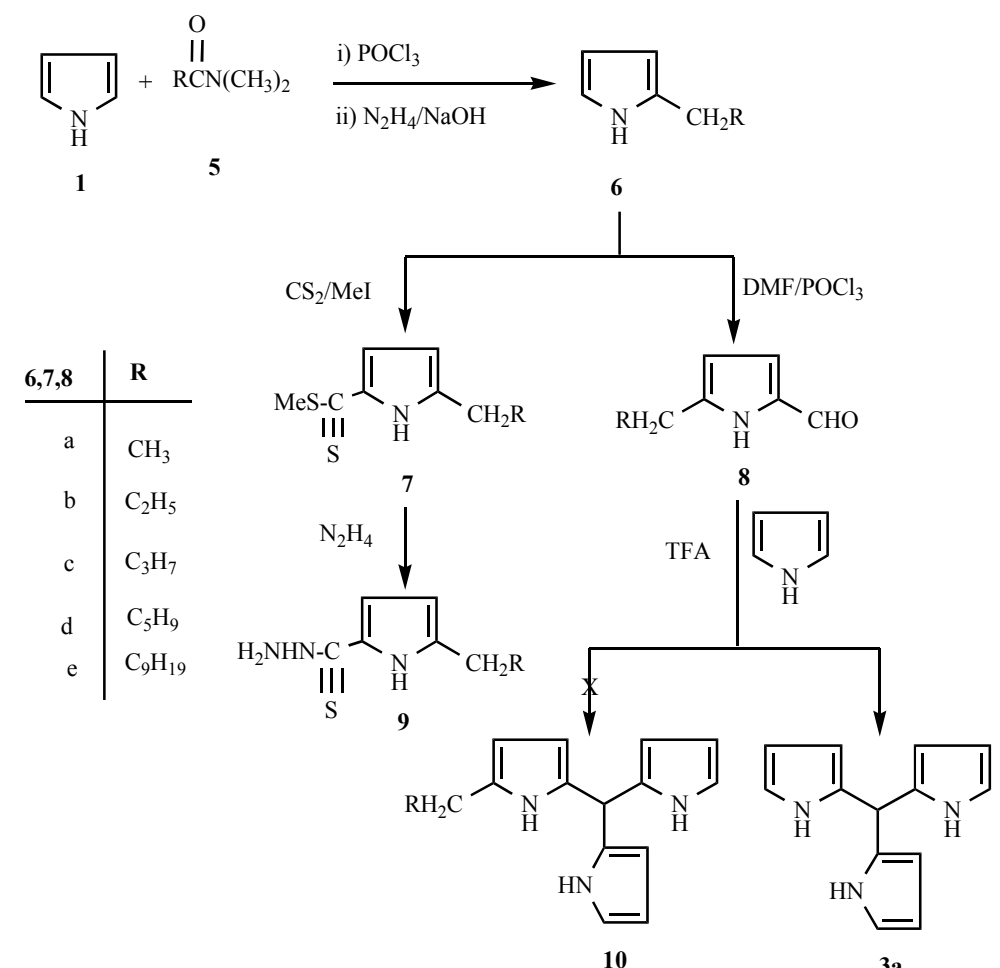

Scheme 2.

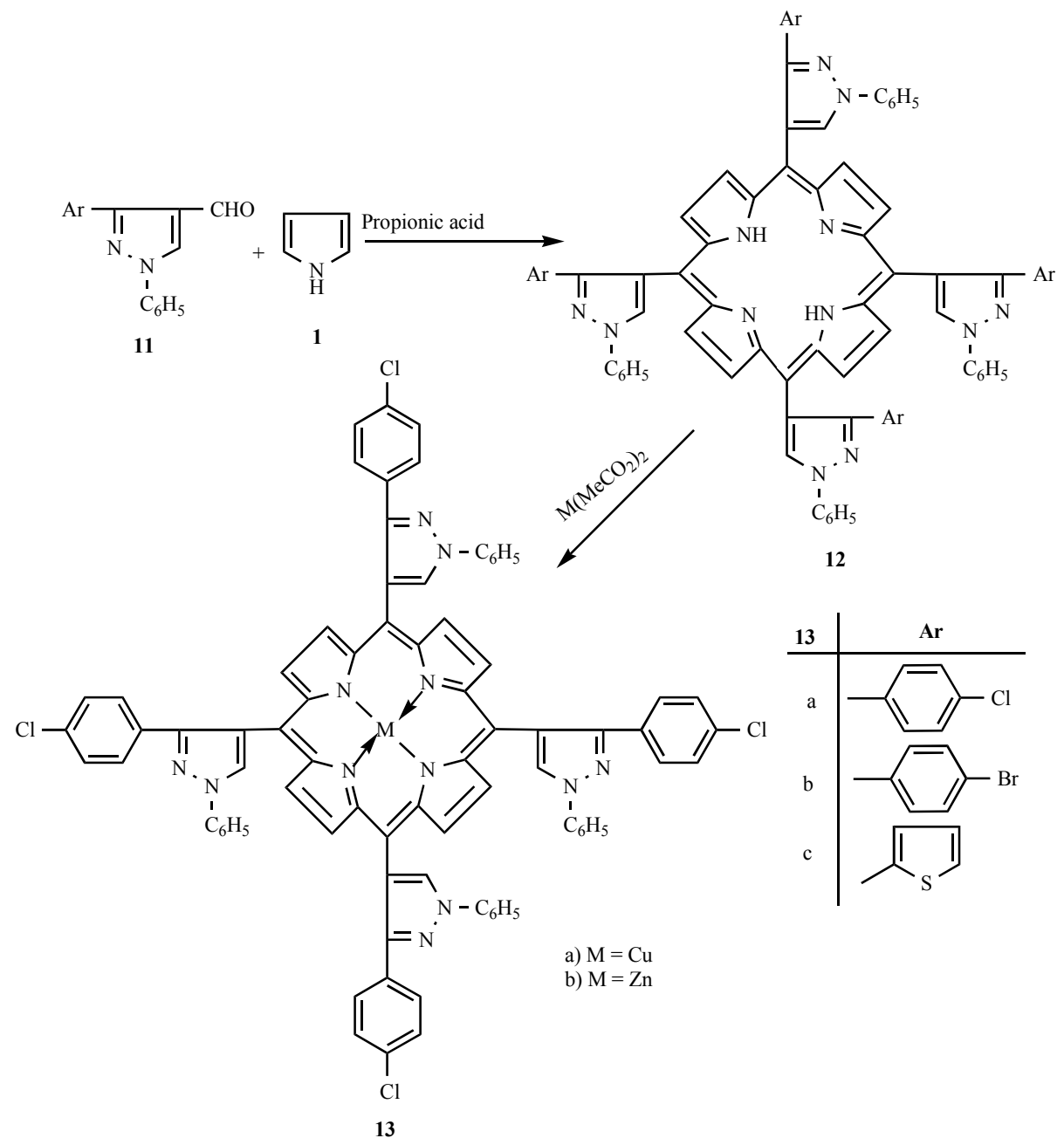

Scheme 3. 
column using a mixture of chloroform-hexane (1:2) as eluent and characterized as $\mathbf{1 2}$. The heterometallic system 13, which involves different metal ions in the chromophoric backbone of the porphyrin was prepared by mixing an equimolar mixture of porphyrin $\mathbf{1 2}$ in dichloromethane and a solution of zinc or copper acetate separately in methanol at room temperature (Scheme 3).

All the synthesized compounds were characterized by their spectroscopic and elemental analyses.

\section{EXPERIMENTAL SECTION}

\section{General Information}

Melting points were determined in an open capillary with a Büchi-530 melting point apparatus and are uncorrected. ${ }^{1} \mathrm{H}$ NMR spectra were recorded on a $200 \mathrm{MHz}$ spectrometer using TMS as internal reference compound (chemical shifts in $\mathrm{ppm}$ ). IR spectra were obtained in $\mathrm{KBr}$ discs. EI-MS were accomplished at $70 \mathrm{eV}$ on a mass spectrometer. Microanalyses $(\mathrm{C}, \mathrm{H}, \mathrm{N})$ were carried out on a Carlo Erba EA-1108 elemental analyzer. TLC was performed on $7 \times 3 \mathrm{~cm}$ precoated silica gel/ neutral alumina plastic plate.

\section{[Di(1H-pyrrol-2-yl)methyl]heteroarenes (3a-m), General Procedure}

To a stirred solution of an aldehyde $(2,1.2 \mathrm{mmol})$ and pyrrole (51.6 mmol), Amberlyst 15 or TFA $(0.38 \mathrm{mmol})$ was added carefully. The reaction mixture was stirred at room temperature for $30 \mathrm{~min}$. Chloroform $(15 \mathrm{~mL})$ was added and the resulting reaction mixture washed with aqueous sodium hydroxide solution (5\%) followed by water. Chloroform layer was collected, dried over $\mathrm{Na}_{2} \mathrm{SO}_{4}$ and evaporated under reduced pressure. Oily crude product obtained was purified by silica gel column chromatography using hexane/ethyl acetate (39:1) as eluent.

\section{2-[Di(1H-pvrrol-2-yl)methyl]-1H-pvrrole (3a)}

White amorphous solid; Mp $128-129^{\circ} \mathrm{C}$ [Lit [22]. $128^{\circ} \mathrm{C}$ ]; yield $75.3 \%$.

\section{3-[Di(1H-pyrrol-2-yl) methyl] pyridine (3b)}

White crystalline solid; Mp $158-160^{\circ} \mathrm{C}$; yield $77.8 \%$; $v_{\max }$ (KBr) $3354 \mathrm{~cm}^{-1}$; $\delta_{\mathrm{H}}\left(200 \mathrm{MHz}, \mathrm{CDCl}_{3}\right) 5.49(\mathrm{~s}, 1 \mathrm{H}, \mathrm{CH})$, 5.87 (dd, J=2.64 Hz, 2H, pyrrolyl), 6.14-6.18 (m, 2H, pyrrolyl), 6.73 (dd, $\mathrm{J}=2.64 \mathrm{~Hz}, 2 \mathrm{H}$, pyrrolyl), $7.27(\mathrm{~m}, 1 \mathrm{H}$, pyridinyl), 7.66 (d, J=8.02 Hz, 1H, pyridinyl), 8.02 (brs, 2H, $\mathrm{NH}), 8.51$ (m, 2H, pyridinyl); m/z (EI) $223\left(\mathrm{M}^{+}\right)$. Anal.Calcd for $\mathrm{C}_{14} \mathrm{H}_{13} \mathrm{~N}_{3}$ : C, 75.3; H, 5.86; N, 18.81 Found: $\mathrm{C}, 75.27 ; \mathrm{H}$, $5.97 ; \mathrm{N}, 18.95$.

\section{3-[Di(1H-pyrrol-2-yl)methyl]-1H-indole (3c)}

White crystalline solid; Mp $128-129^{\circ} \mathrm{C}$; yield $26.6 \%$; $v_{\max }$ $(\mathrm{KBr}) 3360 \mathrm{~cm}^{-1} ; \delta_{\mathrm{H}}\left(200 \mathrm{MHz}, \mathrm{CDCl}_{3}\right) 5.56(\mathrm{~s}, 1 \mathrm{H}, \mathrm{CH})$, 5.84-5.89 (m, 1H, indolyl), 6.02-6.05 (m, 3H, indolyl, pyrrolyl), 6.15-6.22 (m, 3H, indolyl, pyrrolyl), 6.25-6.26 (m, 3H, indolyl, pyrrolyl), 6.81-6.85 (m, 1H, indolyl), 7.95 (brs, 1H, $\mathrm{NH}), 8.02$ (brs, $2 \mathrm{H}, \mathrm{NH}) ; \mathrm{m} / \mathrm{z}(\mathrm{EI}) 261\left(\mathrm{M}^{+}\right)$. Anal.Calcd for $\mathrm{C}_{17} \mathrm{H}_{15} \mathrm{~N}_{3}$ : C, 78.13; H, 5.78; N, 16.07 Found: C, 78.20; H, $5.87 ; \mathrm{N}, 16.21$.

\section{4-[Di(1H-pyrrol-2-yl)methyl]-1,3-diphenyl-1H-pyrazole} $\underline{(3 d)}$

White solid, Mp $130-131^{\circ} \mathrm{C}$; yield $82.7 \%$; $v_{\max }(\mathrm{KBr})$ $3372 \mathrm{~cm}^{-1} ; \delta_{\mathrm{H}}\left(200 \mathrm{MHz}, \mathrm{CDCl}_{3}\right) 5.57(\mathrm{~s}, 1 \mathrm{H}, \mathrm{CH}), 5.59-6.0$ (m, 2H, pyrrolyl), 6.10-6.19 (m, 2H, pyrrolyl), 6.69-6.70 (m, 2H, pyrrolyl), 7.29-7.38 (m, 4H, Rah), 7.57-7.61 (m, 3H, ArH), 7.66-7.73 (m, 4H, ArH), 8.0 (brs, 2H, NH); m/z (FAB) $365\left(\mathrm{M}^{+}+1\right)$. Anal.Calcd for $\mathrm{C}_{24} \mathrm{H}_{20} \mathrm{~N}_{4}$ : C, 79.10; $\mathrm{H}$, 5.53; N, 15.37 Found: C, 79.21; H, 5.62; N, 15.49.

\section{4-[Di(1H-pyrrol-2-yl)methyll-3-(4-methylphenyl)-1-phenyl- 1H-pyrazole (3e)}

White powder; Mp $158-159^{\circ} \mathrm{C}$; yield $80 \%$; $v_{\max }(\mathrm{KBr})$ $3390 \mathrm{~cm}^{-1} ; \delta_{\mathrm{H}}\left(200 \mathrm{MHz}, \mathrm{CDCl}_{3}\right) 2.36\left(\mathrm{~s}, 3 \mathrm{H}, \mathrm{CH}_{3}\right), 5.56(\mathrm{~s}$, $1 \mathrm{H}, \mathrm{CH}), 5.59-6.0$ (m, 2H, pyrrolyl), 6.14-6.18 (m, 2H, pyrrolyl), 6.68-6.71 (m, 2H, pyrrolyl), 7.16-7.28 (m, 3H, ArH), 7.38-7.50 (m, 4H, ArH), 7.68-7.73 (m, 3H, ArH), 7.99 (brs, $2 \mathrm{H}, \mathrm{NH}) ; \mathrm{m} / \mathrm{z}(\mathrm{FAB}) 379\left(\mathrm{M}^{+}+1\right)$. Anal.Calcd for $\mathrm{C}_{25} \mathrm{H}_{22} \mathrm{~N}_{4}$ : C, 79.34; H, 5.86; N, 14.80 Found: C, 79.47; H, 5.95; N, 15.02 .

\section{4-[Di(1H-pyrrol-2-yl)methyl]-3-[4-methoxyphenyl]-1- phenvl-1H-pvrazole (3f)}

White amorphous solid; Mp $168-169^{\circ} \mathrm{C}$; yield $88 \%$; $v_{\max }$ $(\mathrm{KBr}) 3381 \mathrm{~cm}^{-1} ; \delta_{\mathrm{H}}\left(200 \mathrm{MHz}, \mathrm{CDCl}_{3}\right) 3.82\left(\mathrm{~s}, 3 \mathrm{H}, \mathrm{OCH}_{3}\right)$, $5.54(\mathrm{~s}, 1 \mathrm{H}, \mathrm{CH}), 5.59-6.0(\mathrm{~m}, 2 \mathrm{H}$, pyrrolyl), 6.15-6.19 (m, 2H, pyrrolyl), 6.69-6.70 (m, 2H, pyrrolyl), 6.98 (d, J=8.8 Hz, 2H, ArH), 7.28-7.53 (m, 5H, ArH), 7.68-7.72 (m, 3H, ArH), 7.98 (brs, 2H, NH); m/z (FAB) $395\left(\mathrm{M}^{+}+1\right)$. Anal.Calcd for $\mathrm{C}_{25} \mathrm{H}_{22} \mathrm{~N}_{4} \mathrm{O}$ : C, 76.12; H, 5.62; N, 14.20 Found: C, 76.25; H, $5.68 ; \mathrm{N}, 14.12$.

\section{4-[Di(1H-pyrrol-2-yl)methyl]-3-[4-fluorophenyl]-1-phenyl- 1H-pvrazole (3g)}

White crystalline solid; Mp $138-139^{\circ} \mathrm{C}$; yield $85 \%$; $v_{\max }$ $(\mathrm{KBr}) 3361 \mathrm{~cm}^{-1} ; \delta_{\mathrm{H}}\left(200 \mathrm{MHz}, \mathrm{CDCl}_{3}\right) 5.51(\mathrm{~s}, 1 \mathrm{H}, \mathrm{CH})$, 5.59-6.0 (m, 2H, pyrrolyl), 6.16-6.18 (m, 2H, pyrrolyl), 6.706.71 (m, 2H, pyrrolyl), 7.01-7.09 (m, 2H, ArH), 7.25-7.58 (m, 5H, ArH), 7.68-7.72 (m, 3H, ArH), 7.98 (brs, 2H, NH); $\mathrm{m} / \mathrm{z}(\mathrm{FAB}) 383 \mathrm{M}^{+}+1$ ). Anal. Calcd for $\mathrm{C}_{25} \mathrm{H}_{19} \mathrm{FN}_{4}$ : C, 75.37; H, 5.01; N, 14.65 Found: C, 75.55; H, 5.20; N, 14.56.

\section{3-[4-Chlorophenyl]-4-[di(1H-pyrrol-2-yl)methyl]-1-phenyl- 1H-pyrazole (3h)}

White powdered solid; Mp $146-147^{\circ} \mathrm{C}$; yield $84 \%$; $v_{\max }$ $(\mathrm{KBr}) 3370 \mathrm{~cm}^{-1} ; \delta_{\mathrm{H}}\left(200 \mathrm{MHz}, \mathrm{CDCl}_{3}\right) 5.52(\mathrm{~s}, 1 \mathrm{H}, \mathrm{CH})$, 5.58-6.04 (m, 2H, pyrrolyl), 6.14-6.19 (m, 2H, pyrrolyl), 6.70-6.71 (m, 2H, pyrrolyl), 7.23-7.59 (m, 7H, ArH), 7.687.71 (m, 3H, ArH), 7.98 (brs, 2H, NH); m/z (FAB) 399 $\left(\mathrm{M}^{+}+1\right)$. Anal.Calcd for $\mathrm{C}_{25} \mathrm{H}_{19} \mathrm{ClN}_{4}: \mathrm{C}, 72.27 ; \mathrm{H}, 4.80 ; \mathrm{N}$, 14.05 Found: C, 72.34; H, 4.95; N, 14.16

\section{3-[4-Bromophenyl]-4-[di(1H-pyrrol-2-yl)methyl]-1-phenyl- 1H-pyrazole (3i)}

White crystalline solid; Mp $148-149^{\circ} \mathrm{C}$; yield $84 \%$; $v_{\max }$ $(\mathrm{KBr}) 3382 \mathrm{~cm}^{-1} ; \delta_{\mathrm{H}}\left(200 \mathrm{MHz}, \mathrm{CDCl}_{3}\right) 5.51(\mathrm{~s}, 1 \mathrm{H}, \mathrm{CH})$, 5.99-6.0 (m, 2H, pyrrolyl), 6.14-6.19 (m, 2H, pyrrolyl), 6.706.71 (m, 2H, pyrrolyl), 7.24-7.30 (m, 2H, ArH), 7.39-7.52 (m, 5H, ArH), 7.67-7.71 (m, 3H, ArH), 7.97 (brs, 2H, NH). $\mathrm{m} / \mathrm{z}$ (FAB) $444\left(\mathrm{M}^{+}+1\right)$. Anal.Calcd for $\mathrm{C}_{25} \mathrm{H}_{19} \mathrm{BrN}_{4}: \mathrm{C}$, 65.02; H, 4.32; N, 12.62 Found: C, 65.23; H, 4.39; N, 12.76. 


\section{4-[Di(1H-pyrrol-2-yl)methyl]-3-(2-thienvl)-1H-pyrazole (3i)}

White crystalline solid; Mp $150-152^{\circ} \mathrm{C}$; yield $89 \%$; $v_{\max }$ $(\mathrm{KBr}) 3386 \mathrm{~cm}^{-1} ; \delta_{\mathrm{H}}\left(200 \mathrm{MHz}, \mathrm{CDCl}_{3}\right) 5.64(\mathrm{~s}, 1 \mathrm{H}, \mathrm{CH})$, 6.01-6.02 (m, 2H, pyrrolyl), 6.15-6.20 (m, 2H, pyrrolyl), 6.70-6.71 (m, 2H, pyrrolyl), 6.69-7.0 (m, 2H, ArH), 7.157.50 (m, 4H, ArH), 7.56-7.75 (m, 3H, ArH), 8.0 (brs, 2H, $\mathrm{NH}) ; \mathrm{m} / \mathrm{z}(\mathrm{FAB}) 371\left(\mathrm{M}^{+}+1\right)$. Anal.Calcd for $\mathrm{C}_{22} \mathrm{H}_{18} \mathrm{~N}_{4} \mathrm{~S}: \mathrm{C}$, 71.32; H, 4.90; N, 15.12 Found: C, 71.40; H, 5.15; N, 15.22.

\section{5-[Di(1H-pyrrol-2-yl)methyll-3-phenylisoxazole (3k)}

White amorphous solid, Mp $120-121^{\circ} \mathrm{C}$, yield $86 \%$; $v_{\max }$ $(\mathrm{KBr}) 3355 \mathrm{~cm}^{-1} ; \delta_{\mathrm{H}}\left(200 \mathrm{MHz}, \mathrm{CDCl}_{3}\right) 5.56(\mathrm{~s}, 1 \mathrm{H}, \mathrm{CH})$, 6.09-6.20 (m, 4H, pyrrolyl), $6.34(\mathrm{~s}, 1 \mathrm{H}$, isoxazolyl), 6.716.74 (m, 2H, pyrrolyl), 7.41-7.44 (m, 3H, ArH), 7.73-7.77 (m, 2H, ArH), 8.20 (brs, 2H, NH); m/z (FAB) $290\left(\mathrm{M}^{+}+1\right)$. Anal.Calcd for $\mathrm{C}_{18} \mathrm{H}_{15} \mathrm{~N}_{3} \mathrm{O}: \mathrm{C}, 74.72 ; \mathrm{H}, 5.23 ; \mathrm{N}, 14.52$ Found: C, 74.75; H, 5.30; N, 14.76.

\section{5-[Di(1H-pyrrol-2-yl)methyl]-3-(4-methylphenyl)isoxazole (3l)}

White powdered solid; Mp $126-127^{\circ} \mathrm{C}$; yield $88 \%$; $v_{\max }$ (KBr) $3360 \mathrm{~cm}^{-1} ; \delta_{\mathrm{H}}\left(200 \mathrm{MHz}, \mathrm{CDCl}_{3}\right) 2.34\left(\mathrm{~s}, 3 \mathrm{H}, \mathrm{CH}_{3}\right)$, $5.69(\mathrm{~s}, 1 \mathrm{H}, \mathrm{CH}), 6.08-6.19(\mathrm{~m}, 4 \mathrm{H}$, pyrrolyl), $6.32(\mathrm{~s}, 1 \mathrm{H}$, isoxazolyl), 6.72-6.75 (m, 2H, pyrrolyl), $7.23(\mathrm{~d}, \mathrm{~J}=8.10 \mathrm{~Hz}$, 2H, ArH), 7.63 (d, J=8.10 Hz, 2H, ArH), 8.21 (brs, 2H, NH); $\mathrm{m} / \mathrm{z}(\mathrm{FAB}) 304\left(\mathrm{M}^{+}+1\right)$. Anal.Calcd for $\mathrm{C}_{19} \mathrm{H}_{17} \mathrm{~N}_{3} \mathrm{O}: \mathrm{C}$, 75.23; H, 5.65; N, 13.85 Found: C, 75.34; H, 5.51; N, 13.93.

\section{3-(4-Chlorophenyl)-5-[di(1H-pyrrol-2-yl)methyl]isoxazole (3m)}

White crystalline solid; $\mathrm{Mp} 75-76^{\circ} \mathrm{C}$; yield $88 \%$; $v_{\max }$ $(\mathrm{KBr}) 3385 \mathrm{~cm}^{-1} ; \delta_{\mathrm{H}}\left(200 \mathrm{MHz}, \mathrm{CDCl}_{3}\right) 5.52(\mathrm{~s}, 1 \mathrm{H}, \mathrm{CH})$, 6.09-6.19 (m, 4H, pyrrolyl), $6.32(\mathrm{~s}, 1 \mathrm{H}$, isoxazolyl), 6.646.74 (m, 2H, pyrrolyl), 7.42 (d, J=8.10 Hz, 2H, ArH), 7.72 (d, J=8.10 Hz, 2H, ArH), 8.21 (brs, 2H, NH); m/z (FAB) 324 $\left(\mathrm{M}^{+}+1\right)$. Anal.Calcd for $\mathrm{C}_{18} \mathrm{H}_{14} \mathrm{ClN}_{3} \mathrm{O}: \mathrm{C}, 66.77 ; \mathrm{H}, 4.36 ; \mathrm{N}$, 12.98 Found: $\mathrm{C}, 66.85 ; \mathrm{H}, 4.54 ; \mathrm{N}, 12.83$.

\section{5-[Di-(5-formyl-1H-pyrrol-2-yl)methyl]-1H-pyrrole-2- carbaldehyde (4a)}

A solution of phosphorous oxychloride $(6.6 \mathrm{~mL})$ in dichloroethane $(3.5 \mathrm{~mL})$ was slowly added to a solution of $N, N$-dimethylformamide in dichloroethane $(2.5 \mathrm{~mL})$ at $0^{\circ} \mathrm{C}$. The solution was allowed to stir at room temperature for half an hour followed by addition of a solution of $3(1.9 \mathrm{mmol})$ in dichloroethane at $0^{\circ} \mathrm{C}$ with continued stirring for an additional $2 \mathrm{~h}$. at room temperature. Sodium acetate $(22.6 \mathrm{mmol}$ in $10 \mathrm{~mL}$ water) was added to the reaction mixture and then refluxed for $20 \mathrm{~min}$. Dichlorethane layer was separated and aqueous layer extracted with $\mathrm{CHCl}_{3}(3 \times 12 \mathrm{~mL})$. The combined organic layer was dried over $\mathrm{Na}_{2} \mathrm{SO}_{4}$, evaporated under reduced pressure. Crude product thus obtained was purified on silica gel column using hexane/ethyl acetate $(3: 2)$ as eluent. Brown powdered solid; $\mathrm{Mp}>250^{\circ} \mathrm{C}$; yield $45 \%$; $v_{\max }$ (KBr) $1655,3336 \mathrm{~cm}^{-1} ; \delta_{\mathrm{H}}\left(200 \mathrm{MHz}, \mathrm{CDCl}_{3}+\mathrm{DMSO}-\mathrm{d}_{6}\right)$ $5.74(\mathrm{~s}, 1 \mathrm{H}, \mathrm{CH}), 6.18$ (dd, J=2.64 Hz, 3H, pyrrolyl), 6.85 (dd, J=2.64 Hz, 3H, pyrrolyl), 9.41 (s, 3H, CHO), 10.64 (brs, $3 \mathrm{H}, \mathrm{NH}) ; \mathrm{m} / \mathrm{z}(\mathrm{EI}) 295\left(\mathrm{M}^{+}\right)$. Anal. Calcd $\mathrm{C}_{16} \mathrm{H}_{13} \mathrm{~N}_{3} \mathrm{O}_{3}: \mathrm{C}$, 65.08; H, 4.43; N, 14.24 Found: C, 65.04; H, 4.40; N, 14.22.
5-[(5-formyl-1H-pyrrol-2-yl)(3-pyridinyl) methyll-1Hpyrrole-2-carbaldehyde (4b)

It was prepared by following the procedure of $4 \mathbf{a}$. Oil; yield 48\%; $v_{\max }$ (neat) $1651,3346 \mathrm{~cm}^{-1} ; \delta_{\mathrm{H}}(200 \mathrm{MHz}$, $\left.\mathrm{CDCl}_{3}\right) 5.61(\mathrm{~s}, 1 \mathrm{H}, \mathrm{CH}), 6.05$ (dd, J=2.64 Hz, 2H, pyrrolyl), 6.88 (dd, $\mathrm{J}=2.64 \mathrm{~Hz}, 2 \mathrm{H}$, pyrrolyl), 7.28-7.32 (m, 1H, pyridinyl), 7.66 (d, J=8.02 Hz, $1 \mathrm{H}$, pyridinyl), 8.53-8.56 (m, 2H, pyridinyl), 9.40 (brs, $2 \mathrm{H}, \mathrm{CHO}), 10.60$ (s, 2H, NH); m/z (EI) $279\left(\mathrm{M}^{+}\right)$.Anal.Calcd for $\mathrm{C}_{16} \mathrm{H}_{13} \mathrm{~N}_{3} \mathrm{O}_{2}$ : C, 68.80; H, 4.79; N, 15.14 Found: C, 68.77; H, 4.68; N, 15.03.

\section{Methyl 5-alkyl-1H-pyrrole-2-carbodithioate (7a-e), General procedure}

Powdered $\mathrm{KOH}(21.05 \mathrm{mmol})$ was stirred in dimethyl sulphoxide $(20 \mathrm{~mL})$ at room temperature for $20 \mathrm{~min}$. Alkylpyrrole $(6,10.52 \mathrm{mmol})$ was added slowly and allowed the reaction mixture to stir at room temperature for $45 \mathrm{~min}$. Carbon disulphide $(21.05 \mathrm{mmol})$ was charged into reaction mixture and again allowed to stir for $3 \mathrm{~h}$. At the end, reaction mixture was diluted with water and extracted with diethyl ether, dried over $\mathrm{CaCl}_{2}$. The crude product obtained was purified on silica gel column using hexane $/ \mathrm{CH}_{2} \mathrm{Cl}_{2}(9: 1)$ eluent.

\section{Methyl 5-ethyl-1H-pyrrole-2-carbodithioate (7a)}

Oil; yield 42.5\%; $v_{\max }$ (neat) $3349 \mathrm{~cm}^{-1} ; \delta_{\mathrm{H}}(200 \mathrm{MHz}$, $\left.\mathrm{CDCl}_{3}\right) 1.28\left(\mathrm{t}, \mathrm{J}=7.60 \mathrm{~Hz}, 3 \mathrm{H}, \mathrm{CH}_{3}\right), 2.70(\mathrm{q}, \mathrm{J}=7.60 \mathrm{~Hz}$, $\left.2 \mathrm{H}, \mathrm{CH}_{2}\right), 2.72\left(\mathrm{~s}, 3 \mathrm{H}, \mathrm{SCH}_{3}\right), 6.08(\mathrm{dd}, \mathrm{J}=2.54 \mathrm{~Hz}, 1 \mathrm{H}$, pyrrolyl), 7.04 (dd, J=2.54 Hz, 1H, pyrrolyl), 9.40 (brs, $1 \mathrm{H}$, $\mathrm{NH}) ; \mathrm{m} / \mathrm{z}(\mathrm{EI}) 185\left(\mathrm{M}^{+}\right)$. Anal.Calcd for $\mathrm{C}_{8} \mathrm{H}_{11} \mathrm{NS}_{2}$ : C, 51.85; H, 5.98; N, 7.55 Found: C, 51.88; H, 5.84; N, 7.46.

\section{Methyl 5-n-propyl-1H-pyrrole-2-carbodithioate (7b)}

Oil; yield $48.3 \% ; v_{\max }$ (neat) $3348 \mathrm{~cm}^{-1} ; \delta_{\mathrm{H}}(200 \mathrm{MHz}$, $\left.\mathrm{CDCl}_{3}\right) 0.97$ (t, J=7.40 Hz, 3H, $\left.\mathrm{CH}_{3}\right), 1.68\left(\mathrm{~m}, 2 \mathrm{H}, \mathrm{CH}_{2}\right)$, $2.58\left(\mathrm{t}, \mathrm{J}=7.60 \mathrm{~Hz}, 2 \mathrm{H}, \mathrm{CH}_{2}\right), 2.72\left(\mathrm{~s}, 3 \mathrm{H}, \mathrm{SCH}_{3}\right), 6.07$ (dd, $\mathrm{J}=2.54 \mathrm{~Hz}, 1 \mathrm{H}$, pyrrolyl), 7.03 (dd, $\mathrm{J}=2.54 \mathrm{~Hz}, 1 \mathrm{H}$, pyrrolyl), 9.55 (brs, $1 \mathrm{H}, \mathrm{NH}) ; \mathrm{m} / \mathrm{z}$ (EI) $199\left(\mathrm{M}^{+}\right)$. Anal.Calcd for $\mathrm{C}_{9} \mathrm{H}_{13} \mathrm{NS}_{2}$ : C, 54.22; H, 6.57; N, 7.02 Found: C, 54.35; H, $6.67 ; \mathrm{N}, 7.12$.

\section{Methyl 5-n-butyl-1H-pyrrole-2-carbodithioate (7c)}

Oil; yield $46.3 \% ; v_{\max }$ (neat) $3340 \mathrm{~cm}^{-1} ; \delta_{\mathrm{H}}(200 \mathrm{MHz}$, $\left.\mathrm{CDCl}_{3}\right) 0.93\left(\mathrm{t}, \mathrm{J}=7.24 \mathrm{~Hz}, 3 \mathrm{H}, \mathrm{CH}_{3}\right), 1.33-1.44(\mathrm{~m}, 2 \mathrm{H}$, $\mathrm{CH}_{2}$ ), 1.56-1.68 (m, 2H, CH2), 2.60 (t, J=7.36 Hz, 2H, $\mathrm{CH}_{2}$ ), 2.72 (s, 3H, $\left.\mathrm{SCH}_{3}\right), 6.07$ (dd, J=2.54 Hz, 1H, pyrrolyl), 7.04 (dd, J=2.54 Hz, 1H, pyrrolyl), 9.55 (brs H, NH); m/z (EI) $213\left(\mathrm{M}^{+}\right)$. Anal.Calcd for $\mathrm{C}_{10} \mathrm{H}_{15} \mathrm{NS}_{2}: \mathrm{C}, 56.29 ; \mathrm{H}, 7.08 ; \mathrm{N}$, 6.56 Found: C, 56.30; H, 7.17; N, 6.70.

\section{Methyl 5-n-hexyl-1H-pyrrole-2-carbodithioate (7d)}

Oil; yield $49.2 \% ; v_{\max }$ (neat) $3348 \mathrm{~cm}^{-1} ; \delta_{\mathrm{H}}(200 \mathrm{MHz}$, $\left.\mathrm{CDCl}_{3}\right) 0.86\left(\mathrm{t}, \mathrm{J}=6.54 \mathrm{~Hz}, 3 \mathrm{H}, \mathrm{CH}_{3}\right), 1.30-1.34(\mathrm{~m}, 4 \mathrm{H}$, $\mathrm{CH}_{2}$ ), 1.56-1.62 (m, 4H, CH2), 2.60 (t, J=7.36 Hz, 2H, $\mathrm{CH}_{2}$ ), $2.72\left(\mathrm{~s}, 3 \mathrm{H}, \mathrm{SCH}_{3}\right), 6.07$ (dd, J=2.54 Hz, 1H, pyrrolyl), 7.04 (dd, J=2.54 Hz, 1H, pyrrolyl), 9.55 (brs, 1H, NH); m/z (EI) $241\left(\mathrm{M}^{+}\right)$. Anal.Calcd for $\mathrm{C}_{12} \mathrm{H}_{19} \mathrm{NS}_{2}$ : C, 59.70; H, 7.93; N, 5.80 Found: C, 59.68; H, 7.81; N, 5.82.

\section{Methyl 5-n-decvl-1H-pvrrole-2-carbodithioate (7e)}

Oil; yield $52 \% ; v_{\max }$ (neat) $3340 \mathrm{~cm}^{-1} ; \delta_{\mathrm{H}}(200 \mathrm{MHz}$, $\left.\mathrm{CDCl}_{3}\right) 0.87\left(\mathrm{t}, \mathrm{J}=7.24 \mathrm{~Hz}, 3 \mathrm{H}, \mathrm{CH}_{3}\right), 1.23-1.26(\mathrm{~m}, 14 \mathrm{H}$, 
$\left.\mathrm{CH}_{2}\right), 1.64-1.68\left(\mathrm{~m}, 2 \mathrm{H}, \mathrm{CH}_{2}\right), 2.59$ (t, J=6.06 Hz, 2H, $\mathrm{CH}_{2}$ ), $2.72\left(\mathrm{~s}, 3 \mathrm{H}, \mathrm{SCH}_{3}\right), 6.06$ (dd, J=2.54 Hz, 1H, pyrrolyl), 7.03 (dd, J=2.54 Hz, 1H, pyrrolyl), 9.54 (brs, 1H, NH); m/z (EI) $279\left(\mathrm{M}^{+}\right)$. Anal.Calcd for $\mathrm{C}_{16} \mathrm{H}_{27} \mathrm{NS}_{2}$ : C, 64.59; H, 9.14; N, 4.70 Found: C, 64.69; H, 9.25; N, 4.78 .

\section{5-Alkyl-1H-pyrrole-2-carbaldehyde (8a-e), General Proce- dure}

A solution of phosphorous oxychloride $(2.92 \mathrm{mmol})$ in dry benzene $(5 \mathrm{~mL})$ was added to a solution of $N, N$ dimethylformamide $(2.68 \mathrm{mmol})$ in dry benzene $(4 \mathrm{~mL})$ at $0^{\circ} \mathrm{C}$ for $30 \mathrm{~min}$. and thereafter stirred at $20^{\circ} \mathrm{C}$ for half an hour. Alkylpyrroles $(6,2.43 \mathrm{mmol})$ in dry benzene $(5 \mathrm{~mL})$ was added slowly at $0^{\circ} \mathrm{C}$ and further stirred for $18 \mathrm{~h}$ at room temperature. The reaction mixture was diluted with ice cold water $(15 \mathrm{~mL})$ neutralized with sodium bicarbonate and $\mathrm{NaOH}$ solution $(40 \%)$ added to adjust $\mathrm{pH} 12$. The alkaline solution was stirred at room temperature for $1.5 \mathrm{~h}$. Benzene phase was separated and aqueous layer extracted with $\mathrm{CHCl}_{3}$ $(3 \times 30 \mathrm{~mL})$. The combined extract was dried over $\mathrm{Na}_{2} \mathrm{SO}_{4}$, evaporated to dryness. The crude product was finally purified on silica gel column using $\mathrm{CH}_{2} \mathrm{Cl}_{2} / \mathrm{Hexane}(1: 1)$ as eluent.

\section{5-Ethyl-1H-pyrrole-2-carbaldehyde (8a)}

White semi solid; yield $77.2 \%$; $v_{\max }$ (neat) 1643,3440 $\mathrm{cm}^{-1} ; \delta_{\mathrm{H}}\left(200 \mathrm{MHz}, \mathrm{CDCl}_{3}\right) 1.28\left(\mathrm{t}, \mathrm{J}=7.60 \mathrm{~Hz}, 3 \mathrm{H}, \mathrm{CH}_{3}\right)$, 2.70 (q, J=7.60 Hz, 2H, $\left.\mathrm{CH}_{2}\right), 6.89(\mathrm{dd}, \mathrm{J}=2.64 \mathrm{~Hz}, 1 \mathrm{H}$, pyrrolyl), 7.04 (dd, J=2.64 Hz, 1H, pyrrolyl), 9.37 (s, 1H, CHO), 9.72 (brs, 1H, NH); m/z (EI) $123\left(\mathrm{M}^{+}\right)$. Anal.Clacd for $\mathrm{C}_{7} \mathrm{H}_{9} \mathrm{NO}$ : $\mathrm{C}, 68.26$; H, 7.37; N, 11.37. Found: C, 68.34; $\mathrm{H}, 7.45 ; \mathrm{N}, 11.48$.

\section{5-n-Propyl-1H-pyrrole-2-carbaldehyde (8b)}

White semi solid; yield $72 \%$; $v_{\max }$ (neat) $1643,3350 \mathrm{~cm}^{-1}$; $\delta_{\mathrm{H}}\left(200 \mathrm{MHz} \mathrm{CDCl}_{3}\right) 0.97\left(\mathrm{t}, \mathrm{J}=7.40 \mathrm{~Hz}, 3 \mathrm{H}, \mathrm{CH}_{3}\right), 1.58-$ $164\left(\mathrm{~m}, 2 \mathrm{H}, \mathrm{CH}_{2}\right), 2.64\left(\mathrm{t}, \mathrm{J}=7.60 \mathrm{~Hz}, 2 \mathrm{H}, \mathrm{CH}_{2}\right), 6.07$ (dd, $\mathrm{J}=2.64 \mathrm{~Hz}, 1 \mathrm{H}$, pyrrolyl), 6.89 (dd, $\mathrm{J}=2.60 \mathrm{~Hz}, 1 \mathrm{H}$, pyrrolyl), 9.36 (s, 1H, CHO), 9.72 (brs, 1H, NH); m/z (EI) $137\left(\mathrm{M}^{+}\right)$. Anal.Calcd for $\mathrm{C}_{8} \mathrm{H}_{11} \mathrm{NO}$ : C, 70.04; H, 8.08; N, 10.21 . Found: C, 70.23; H, 8.14; N, 10.13.

\section{5-n-Butyl-1H-pyrrole-2-carbaldehyde (8c)}

White semi solid; yield 69.7\%; $v_{\max }$ (neat) 1642, 3345 $\mathrm{cm}^{-1} ; \delta_{\mathrm{H}}\left(200 \mathrm{MHz}, \mathrm{CDCl}_{3}\right) 0.92\left(\mathrm{t}, \mathrm{J}=7.24 \mathrm{~Hz}, 3 \mathrm{H}, \mathrm{CH}_{3}\right)$, 1.28-1.46 (m, 2H, $\left.\mathrm{CH}_{2}\right), 1.57-1.72\left(\mathrm{~m}, 2 \mathrm{H}, \mathrm{CH}_{2}\right), 2.65(\mathrm{t}$, $\left.\mathrm{J}=7.36 \mathrm{~Hz}, 2 \mathrm{H}, \mathrm{CH}_{2}\right), 6.07$ (dd, $\mathrm{J}=2.64 \mathrm{~Hz}, 1 \mathrm{H}$, pyrrolyl), 6.88 (dd, J=2.64 Hz, 1H, pyrrolyl), 9.37 (s, 1H, CHO), 9.70 (brs, $1 \mathrm{H}, \mathrm{NH}) ; \mathrm{m} / \mathrm{z}(\mathrm{EI}) 151\left(\mathrm{M}^{+}\right)$. Anal.Calcd for $\mathrm{C}_{9} \mathrm{H}_{13} \mathrm{NO}$ : C, 71.48; H, 8.66; N, 9.26 Found: C, 71.38; H, 8.60; N, 9.35.

\section{5-n-Hexyl-1H-pyrrole-2-carbaldehyde (8d)}

White semi solid; yield 63.3\%; $v_{\max }$ (neat) 1643, 3348 $\mathrm{cm}^{-1} ; \delta_{\mathrm{H}}\left(200 \mathrm{MHz}, \mathrm{CDCl}_{3}\right) 0.88\left(\mathrm{t}, \mathrm{J}=6.54 \mathrm{~Hz}, 3 \mathrm{H}, \mathrm{CH}_{3}\right)$, 1.25-1.38 (m, 6H, $\left.\mathrm{CH}_{2}\right), 1.57-1.65\left(\mathrm{~m}, 2 \mathrm{H}, \mathrm{CH}_{2}\right), 2.65(\mathrm{t}$, $\left.\mathrm{J}=7.60 \mathrm{~Hz}, 2 \mathrm{H}, \mathrm{CH}_{2}\right), 6.07$ (dd, J=2.64 Hz, 1H, pyrrolyl), 6.88 (dd, J=2.64 Hz, 1H, pyrrolyl), 9.36 (s, 1H, CHO), 9.56 (brs, $1 \mathrm{H}, \mathrm{NH}) ; \mathrm{m} / \mathrm{z}(\mathrm{EI}) 179\left(\mathrm{M}^{+}\right)$. Anal.Calcd for $\mathrm{C}_{11} \mathrm{H}_{17} \mathrm{NO}$ : C, 73.70; H, 9.55; N, 7.81 Found: C, 73.82; H, 9.63; N, 7.87.

\section{5-n-Decyl-1H-pyrrole-2-carbaldehyde (8e)}

White crystalline solid; Mp $40-41^{0} \mathrm{C}$; yield $71 \%$; $v_{\max }$ (KBr) $1642,3341 \mathrm{~cm}^{-1} ; \delta_{\mathrm{H}}\left(200 \mathrm{MHz}, \mathrm{CDCl}_{3}\right) 0.87(\mathrm{t}$,
$\left.\mathrm{J}=7.24 \mathrm{~Hz}, 3 \mathrm{H}, \mathrm{CH}_{3}\right), 1.23-1.26\left(\mathrm{~m}, 12 \mathrm{H}, \mathrm{CH}_{2}\right), 1.62-1.68$ $\left(\mathrm{m}, 4 \mathrm{H}, \mathrm{CH}_{2}\right) 2.64\left(\mathrm{t}, \mathrm{J}=7.46 \mathrm{~Hz}, 2 \mathrm{H}, \mathrm{CH}_{2}\right), 6.06(\mathrm{dd}, \mathrm{J}=2.64$ $\mathrm{Hz}, 1 \mathrm{H}$, pyrrolyl), 6.87 (dd, J=2.64 Hz, 1H, pyrrolyl), 9.30 (s, 1H, CHO), 9.54 (brs, 1H, NH); m/z (EI) $235\left(\mathrm{M}^{+}\right)$. Anal.Calcd for $\mathrm{C}_{15} \mathrm{H}_{25} \mathrm{NO}: \mathrm{C}, 76.54 ; \mathrm{H}, 10.70 ; \mathrm{N}, 5.95$ Found: C, 76.48; H, 10.83; N, 6.01.

\section{5-Hexyl-1H-pyrrole-2-carbothiohydrazide (9)}

White crystalline solid; $\mathrm{Mp}>250^{\circ} \mathrm{C}$; yield $45.5 \%$; $v_{\max }$ (KBr) $3402 \mathrm{~cm}^{-1} ; \delta_{\mathrm{H}}\left(200 \mathrm{MHz}, \mathrm{CDCl}_{3}\right) 0.88(\mathrm{t}, \mathrm{J}=6.72 \mathrm{~Hz}$, $\left.3 \mathrm{H}, \mathrm{CH}_{3}\right), 1.30-1.31\left(\mathrm{~m}, 4 \mathrm{H}, \mathrm{CH}_{2}\right), 1.57-1.59\left(\mathrm{~m}, 4 \mathrm{H}, \mathrm{CH}_{2}\right)$, 2.59 (t, J=7.38 Hz, 2H, $\mathrm{CH}_{2}$ ), 5.99 (dd, J=2.50 Hz, 1H, pyrrolyl), 6.48 (dd, J=2.50 Hz, 1H, pyrrolyl), 8.32 (brs, $2 \mathrm{H}$, $\mathrm{NH}), 9.45$ (brs, 1H, NH); m/z (EI) $225\left(\mathrm{M}^{+}\right)$. Anal.Calcd $\mathrm{C}_{11} \mathrm{H}_{19} \mathrm{~N}_{3} \mathrm{~S}$ : C, 58.63; H, 8.50; N, 18.65 Found: C, 58.49; H, $8.62 ; \mathrm{N}, 18.72$.

\section{5,10,15,20-Tetra-(1,3-diaryl-1H-pyrazol-4-yl)-porphyrin (12a-c), General Procedure}

A mixture of 1,3-diaryl-4-formylpyrazoles (12, 3.53 $\mathrm{mmol})$ and pyrrole $(\mathbf{1}, 4.48 \mathrm{mmol})$ was stirred in propionic acid $(15 \mathrm{~mL})$ at $135-140^{\circ} \mathrm{C}$ under nitrogen atmosphere for 5 h. Reaction mixture was diluted with distilled water, solid product separated was filtered, washed with excess of water and purified on neutral alumina column using hexane $/ \mathrm{CHCl}_{3}$ $(3: 2)$ as eluent.

\section{5,10,15,20-Tetrakis-[3-(4-chlorophenyl)-1-phenyl-1H- pyrazol-4-yll porphyrin (12a)}

Brown amorphous solid; $\mathrm{Mp}>250^{\circ} \mathrm{C}$; yield $38 \%$; $v_{\max }$ (KBr) $3422 \mathrm{~cm}^{-1} ; \delta_{\mathrm{H}}\left(200 \mathrm{MHz}, \mathrm{CDCl}_{3}\right)$ 6.67-6.80 (m, 8H, $\mathrm{ArH})$, 7.09-7.29 (m, 4H, ArH), 7.33-7.44 (m, 8H, ArH), 7.51-7.63 (m, 8H, ArH), 8.08 (d, J=7.46H, 8H, ArH), 8.518.62 (m, 4H, ArH), 8.65 (brs, 2H, NH), 8.99-9.03 (m, 8H, ArH); m/z (FAB) $1319\left(\mathrm{M}^{+}+1\right), 1320\left(\mathrm{M}^{+}+2\right), 1321\left(\mathrm{M}^{+}+3\right)$. Anal.Calcd for $\mathrm{C}_{80} \mathrm{H}_{50} \mathrm{Cl}_{4} \mathrm{~N}_{12}: \mathrm{C}, 72.73 ; \mathrm{H}, 3.81 ; \mathrm{N}, 12.72$ Found: C, 72.67; H, 3.97; N, 12.69.

\section{5,10,15,20-Tetrakis-[3-(4-bromophenyl)-1-phenyl-1H- pyrazol-4-yll porphyrin (12b)}

Brown amorphous powder; $\mathrm{Mp}>250^{\circ} \mathrm{C}$; yield $35 \%$; $v_{\max }$ (KBr) $3427 \mathrm{~cm}^{-1} ; \delta_{\mathrm{H}}\left(200 \mathrm{MHz}, \mathrm{CDCl}_{3}\right)$ 6.85-6.96 (m, 8H, $\mathrm{ArH})$, 7.17-7.31 (m, 4H, ArH), 7.35-7.7.41 (m, 8H, ArH), 7.55-7.59 (m, 8H, ArH), 8.02-8.10 (m, 8H, ArH), 8.68-8.76 (m, 4H, ArH), 8.79 (brs, 2H, NH), 8.98-9.04 (m, 8H, ArH); $\mathrm{m} / \mathrm{z} \quad(\mathrm{FAB}) 1499\left(\mathrm{M}^{+}+1\right), 1500\left(\mathrm{M}^{+}+2\right), 1501 \quad\left(\mathrm{M}^{+}+3\right)$. Anal.Calcd for $\mathrm{C}_{80} \mathrm{H}_{50} \mathrm{Br}_{4} \mathrm{~N}_{12}$ : C, 64.10; H, 3.36; N, 11.21 Found: C, 64.17; H, 3.50; N, 11.10.

\section{5,10,15,20-Tetrakis-[3-(2-thienyl)-1-phenyl-1H-pyrazol-4- yll porphyrin (12c)}

Gray amorphous solid; $\mathrm{Mp}>250^{\circ} \mathrm{C}$; yield $29 \%$; $v_{\max }$ (KBr) $3423 \mathrm{~cm}^{-1} ; \delta_{\mathrm{H}}\left(200 \mathrm{MHz}, \mathrm{CDCl}_{3}\right) 6.05-6.42(\mathrm{~m}, 12 \mathrm{H}$, ArH), 6.80-6.87 (m, 8H, ArH), 7.36-7.43 (m, 4H, ArH), 7.55-7.62 (m, 4H, ArH), 8.00-8.09 (m, 4H, ArH), 8.44 (brs, 2H, NH), 8.51-8.53 (m, 2H, ArH), 8.64-8.67 (m, 4H, ArH), 8.83-8.85 (m, 2H, NH), 9.07 (s, 4H, ArH); m/z (FAB) 1207 $\left(\mathrm{M}^{+}+1\right), 1208\left(\mathrm{M}^{+}+2\right)$. Anal.Calcd $\mathrm{C}_{72} \mathrm{H}_{46} \mathrm{~N}_{12} \mathrm{~S}_{4}: \mathrm{C}, 71.62 ; \mathrm{H}$, 3.84; N, 13.92 Found: C, 71.58; H, 3.98; N, 13.83 . 


\section{Cu(II)-5,10,15,20-Tetrakis-[3-(4-chlorophenvl)-1-phenyl- 1H-pyrazol-4-yl] porphyrin (13a)}

A solution of $(12 \mathrm{a} 0.05 \mathrm{mmol})$ in $\mathrm{CH}_{2} \mathrm{Cl}_{2}(6 \mathrm{~mL})$ was added slowly to a solution of cupric acetate monohydrate $(0.27 \mathrm{mmol})$ in methanol $(10 \mathrm{~mL})$ at room temperature. The solution was stirred for $1 \mathrm{~h}$. The precipitate obtained was filtered, washed with excess of methanol. Brown amorphous solid; $\mathrm{mp}>250^{0} \mathrm{C}$; yield $91 \% ; v_{\max }(\mathrm{KBr}) 3422 \mathrm{~cm}^{-1} ; \delta_{\mathrm{H}}(200$ $\mathrm{MHz}, \mathrm{CDCl}_{3}$ ) 6.71-6.90 (m, 8H, ArH), 7.26-7.37 (m, $12 \mathrm{H}$, ArH), 7.54-7.60 (m, 8H, ArH), 7.96-8.05 (m, 8H, ArH), 8.43-8.57 (m, 4H, ArH), 8.63-8.68 (m, 4H, ArH), 8.99-9.04 $(\mathrm{m}, 4 \mathrm{H}, \quad \mathrm{ArH}) ; \mathrm{m} / \mathrm{z}(\mathrm{FAB}) 1382\left(\mathrm{M}^{+}+1\right), 1383\left(\mathrm{M}^{+}+2\right)$. Anal.Calcd for $\mathrm{C}_{80} \mathrm{H}_{48} \mathrm{Cl}_{4} \mathrm{~N}_{12} \mathrm{Cu}: \mathrm{C}, 69.49 ; \mathrm{H}, 3.50 ; \mathrm{N}, 11.63$ Found: C, 69.68; H, 3.69; N, 12.16 .

\section{Zn(II)-5,10,15,20-Tetrakis-/3-(4-chlorophenvl)-1-phenvl- 1H-pvrazol-4-vll porphyrin (13b)}

To a solution of zinc chloride $(0.0906 \mathrm{mmol})$ and sodium acetate $(0.0906 \mathrm{mmol})$ in methanol $(15 \mathrm{~mL})$, a solution of porphyrin (12a, $0.015 \mathrm{mmol})$ in $\mathrm{CH}_{2} \mathrm{Cl}_{2}(5 \mathrm{~mL})$ was added at room temperature. The reaction mixture was stirred for $1 \mathrm{~h}$. and filtered the precipitated solid, washed with methanol.

Brown amorphous solid; $\mathrm{Mp}>250^{\circ} \mathrm{C}$; yield $93 \% ; v_{\max }$ (KBr) $3422 \mathrm{~cm}^{-1} ; \delta_{\mathrm{H}}\left(200 \mathrm{MHz}, \mathrm{CDCl}_{3}\right) 6.64-6.80(\mathrm{~m}, 8 \mathrm{H}$, ArH), 7.18-7.29 (m, 4H, ArH), 7.33-7.44 (m, 8H, ArH), 7.56-7.63 (t, J=7.76 Hz, 8H, ArH), 8.09 (d, J=9.03 Hz, 8H, ArH), 8.62-8.74 (m, 4H, ArH), 8.99-9.09 (m, 4H, ArH), 9.12 (s, 4H, ArH); m/z (FAB) $1384\left(\mathrm{M}^{+}+1\right), 1385\left(\mathrm{M}^{+}+2\right)$. Anal.Calcd for $\mathrm{C}_{80} \mathrm{H}_{48} \mathrm{Cl}_{4} \mathrm{~N}_{12} \mathrm{Zn}$ : C, 69.40; H, 3.49; N, 12.14 Found: C, 69.52; H, 3.66; N, 12.25 .

\section{ACKNOWLEDGEMENTS}

Farhanullah is thankful to CSIR for financial support as senior research fellow. VJR thanks Dr. S. Batra for providing 3-aryl-5-formylisoxazoles.

\section{REFERENCES}

[1] Lenninger, A. L.; Nelson, D. L.; Cox, M. M. Principles of Biochemistry; Worth Publishers: New York, 1994.

[2] (a) Smith, K.M. Ed. Pophyrins and Metalloporphyrins; Elsevier: Amsterdam, 1975; (b) Reek, J. N. H.; Rowan, A. E.; de Gelder, R.; Beursken, P.T.; Crossley, M. J.; De Feuter, S.; de Scryver, F.; Nolte, R. J. M. Angew. Chem. Int. Ed. Engl. 1997, 36, 361.
[3] (a) Dolphin, D. The Porphyrins; Academic Press: New York, 1978; Vol. 8. (b) Sheldon, R. A. Metalloporphyrins in Catalytic Oxidation; Marcel Dekker, New York. 1994; (c) Bonnet, R. Chem. Soc. Rev. 1995, 19; (d) Peng, J, B.; Lawrie, G. A.; Barnes, G. T. Gentle, I. R.; Crossley, F. M. J.; Huang, Z. Langmuir 2000, 16, 7051.

[4] (a) Becher, J.; Brimert, T.; Jeppesen, J. O.; Pedersen, J. Z.; Zubarev, R.; Bjornholm, T.; Reitzel, N.; Jensen, T. R.; Kjaer, K.; Levillian, E. Angew. Chem. Int. Ed. 2001, 40, 2497; (b) Adler, A. D. N. Y. Ann. Acad. Sci. 1973, 206; (c) Sharghi, H.; Naeimi, H. J. Chem. Res. 1999, 310; (d) Mansuy, D. Coord. Chem. Rev. 1993 129; (e) Liang, Y.; Chang, C.K. Tetrahedron Lett. 1995, 36, 3817.

[5] (a) Ruthemund, P. J. Am. Chem. Soc. 1939, 61, 2912; (b) Adler, A. D.; Longo, F. R.; Kampas, F.; Kim, J. J. Inorg. Nucl. Chem. 1970, 32,2443 .

[6] (a) Sternberg, E. D.; Dolphin, D.; Bruckner, C. Tetrahedron 1998 54,4151

[7] Lindsey, J. S. In Metalloporphyrins Catalyzed Oxidations; Kluwer Academic Publishers: The Netherlands, 1994, p. 49.

[8] Jasat, A.; Dolphin, D. Chem. Rev. 1997, 22677.

[9] Nagarkatti, J. P.; Ashley, K. R. Synthesis 1974, 97, 186.

[10] Casiraghi, G.; Cornia, M.; Rassu, G.; Del Sante, C.; Spanu, P. Tetrahedron 1992, 48, 5619.

[11] Hammel, D.; Erk, P.; Schuler, B.; Heinze, J.; Müllen, K. Adv. Mater. 1992, 4, 737

[12] Boyle, R. W.; Xie, L. Y.; Dolphin, D. Tetrahedron Lett. 1994, 35 , 5377.

[13] Casiraghi, G.; Cornia, M.; Zanardi, F.; Rassu, G.; Ragg, E.; Bortohini, R. J. Org. Chem. 1994, 59, 1801

[14] Litter, B. J; Miller, M.A.; Hung, C. H.; Wagner, R. W.; O’Shea, D. F. Boyle, P. D.; Lindsey, J. S. J. Org. Chem. 1999, 64, 1391.

[15] Vigmond, S. J.; Chang, M. C.; Kallury, K. M. R.; Thompson, M. Tetrahedron Lett. 1994, 35, 2455.

[16] Wallace, D. M.; Smith, K. M. Tetrahedron Lett. 1990, 31, 7265

[17] Setsune, J.; Hashimoto, M. J. Chem. Soc. Chem. Commun. 1994, 657.

[18] (a) Longo, F.R.; Finarelli, J. D.; Kim, J. J. Heterocycl. Chem. 1969 6, 927; (b) Adler, A. D.; Longo, F. R.; Finarelli, J. D.; Goldmacher, J.; Assour, J.; Korsakoff, L. J. Org. Chem. 1967, 32, 476.

[19] (a) Lindsey, J. S.; Hsu, H. C.; Schreiman, I. C. Tetrahedron Lett. 1986, 27, 49969; (b) Lindsey, J. S.; Schreiman, I. C.; Hsu, H. C.; Kearney, P. C.; Marguerettaz, A. M. J. Org. Chem. 1987, 52, 827.

[20] Garrido, D. O. A.; Buldain, G.; Frydman, B. J. Org. Chem. 1984 49, 2619 .

[21] (a) Rainer, G.; Karuger, U.; Klemn, K. Arznemittel- Forschung 1981, 31, 649; (b) Reidel, R. Arznemittel- Forschung 1981, 31 , 655.

[22] Rees, C.B.; Yan, H. Y. Tetrahedron Lett. 2001, 42, 5545.

Received: March 24, 2009

(C) Farhanullah and Ram; Licensee Bentham Open.

This is an open access article licensed under the terms of the Creative Commons Attribution Non-Commercial License (http://creativecommons.org/licenses/by-nc/3.0/) which permits unrestricted, non-commercial use, distribution and reproduction in any medium, provided the work is properly cited. 